\title{
Practical modeling of non-stationary temperature fields of fiber-optic gyroscopes in space flight conditions
}

\author{
$V M$ Pankratov $^{1}, M A$ Barulina ${ }^{1, *}, A V$ Golikov $^{1}, E V$ Pankratova $^{1}$, and $M V$ Efremov $^{2}$ \\ ${ }^{1}$ Institute of precision mechanics and control problems, Russian Academy of Sciences, Saratov, \\ 410028, Russia \\ ${ }^{2}$ Research and production enterprise "Antares", Saratov, 410600, Russia
}

\begin{abstract}
Based on the mathematical model presented in the first part of this paper, specialized software was created and computer modeling of nonstationary inhomogeneous temperature fields in a fiber-optic gyroscope was performed. The simulation aimed to analyze the temperature distribution in the gyroscope structural elements, especially in the fiber-optic coil and the electronics unit, since temperature fluctuations in them are one of the main sources of device errors. To achieve this goal, a three-dimensional unsteady field was calculated in a fiber-optic gyroscope. Based on the results of computer modeling, comparative data on the temperature distribution in the gyroscope structural elements on earth and orbital flight conditions, and the degree of influence of space and zero-gravity conditions are obtained.
\end{abstract}

\section{Introduction}

In the first part of the work the main research tasks were formulated, a thermal and mathematical model of a fiber-optic gyroscope (FOG) was constructed. The structural elements of FOG were divided into thermal elementary volumes. Two thermal modes (on earth and in outer space) are defined, according to which the parameters of the external environment and the low of temperature disturbances are set.

All formulas and relations [1-3] that form the basis of the mathematical model for calculating the non-stationary inhomogeneous temperature field of a FOG are implemented in specialized software in $\mathrm{C}++$. The software has the functions for calculating, processing model parameters, and initial data, functions for dynamic visualization of thermal processes, which occur in FOG.

The study was carried out in several stages, which simulated the earth's operating conditions, space flight conditions, constant and variable temperature effects to the FOG.

\footnotetext{
* Corresponding author: marina@barulina.ru
} 


\section{Numerical experiments}

\subsection{Earth conditions}

At the first stage, a series of computational experiments were carried out, where earthly conditions (the 1 st thermal mode) were considered as operating conditions.

Figure 1 shows a color diagram of the FOG temperature fields, based on the results of mathematical modeling of the 1-st thermal mode [4] in the moment of the transient process when the ambient temperature increases from the initial $\left(20^{\circ} \mathrm{C}\right)$ up to $44-45^{\circ} \mathrm{C}$ in steadystate mode.

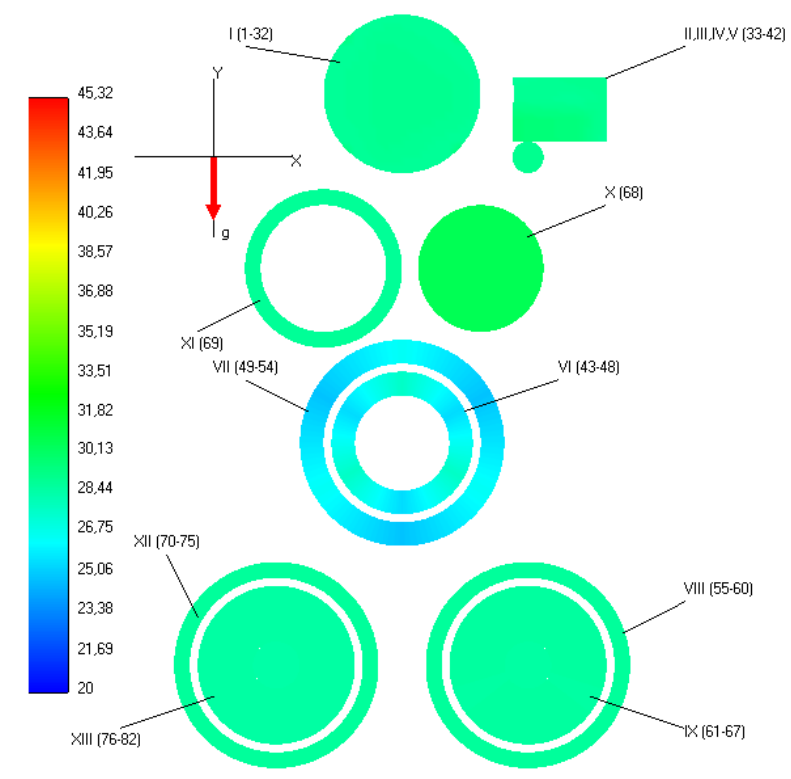

Fig. 1. FOG temperature fields under normal ambient pressure and normal gravity at time $t=20 \mathrm{~min}$ (transient process) I - cover (1-32); II, III,IV,V - electronic boards and superluminescent diode (3342); VI - ring for fiber-optic coil (43-48); VII - fiber-optic coil (49-54); VIII - side of the base (5560 ); IX - the bottom of base (61-67); X- heat shield (68); XI- plate (69); XII - side of the glass (70$75)$; XIII - bottom of the glass (76-82).

The diagram shows the inhomogeneous temperature distribution over the volume of the fiberoptic coil (VII), which can lead to short-term thermal drift of the device. The inhomogeneous temperature distribution occurs during a transient process, for example, when the device is switched on, or in the case of the ambient temperature changing over time according to some law. The value of temperature gradients in the fiber coil and current temperature at all design points of the FOG thermal model in the 1-st thermal mode can be estimated using the graphs below (Figure 2). 


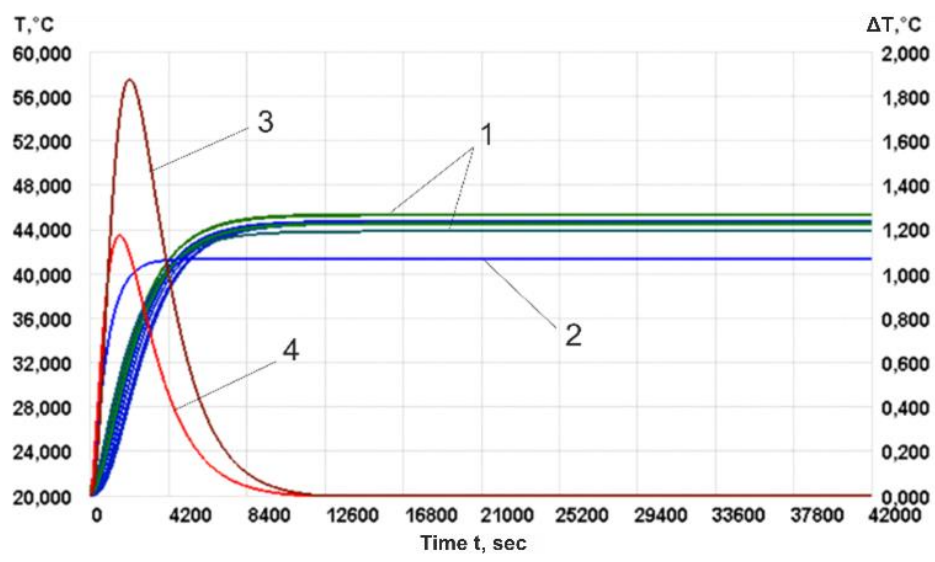

Fig. 2. Current temperatures $T_{i}$ of the FOG's elements and thermal circumferential $\Delta T_{\psi}$ and radial $\Delta T_{R}$ gradients in fiber-optic structure in case of normal environmental pressure and normal gravity 1 temperature of elements $T_{i}(1-82) \mathrm{FOG} ; 2$ - environmental temperature; 3 - circumferential temperature drop in fiber-optic coil of the FOG $\Delta T \psi ; 4$ - radial drop of temperature in fiber-optic coil of the FOG $\Delta T_{R}$.

As seen from Figure 2, the maximum overheating of the device elements above the ambient temperature does not exceed $\Delta T_{\max }=4^{\circ} \mathrm{C}$ under conditions of normal ambient pressure and normal gravity.

Such maximum overheating occurs on the electronics board in the areas of heat sources with the maximum heat output power.

The circumferential and radial temperature gradients in the zone of the optical fiber coil of the FOG in the transition process can take maximum values at the level of $\Delta T_{\psi}=1,9{ }^{\circ} \mathrm{C}$ and $\Delta T_{\mathrm{R}}=1,2{ }^{\circ} \mathrm{C}$ respectively. In steady-state mode, the temperature in the fiber is equalized and the gradients are close to zero.

In conditions of normal atmospheric pressure and gravity, it is of interest to qualitatively and quantitatively assess the effect of its orientation relative to $g$ on the temperature characteristics of the FOG. For this purpose, the proposed model varied the orientation angle $\varphi$ relative to the gravity vector. The results of the study are shown in Figure 3.

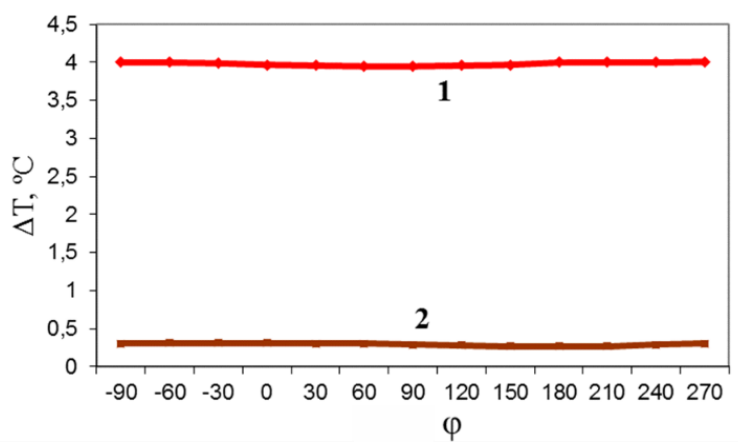

Fig. 3. Dependences of the FOG's temperature characteristics on its orientation relative to $g$ : 1 $\Delta T_{\max }(\varphi), 2-\Delta T_{\psi}(\varphi)$.

The Figure 3 shows that the thermal situation in the FOG under normal atmospheric pressure and gravity is almost independent of the orientation of the FOG relative to $g$.

In general, the coefficients of free-convective heat transfer under normal atmospheric pressure and gravity from the FOG elements to the environment depend on the orientation of 
the device relative to $g$. However, according to the calculation results, the total heat transfer coefficient to the environment in the position $\varphi=90^{\circ}$ ("upside-down") it differs only by $\approx 2 \%$ from the value in the pase position $\varphi=-90^{\circ}$. This fact can be explained that when the FOG is turned by $180^{\circ}$ the decrease in heat transfer from some elements is compensated by the increase in heat transfer from other elements.

An important question that arises and should be resolved is "how the temperature situation in the device will change in space flight under vacuum and weightlessness conditions?"

\subsection{The conditions of outer space}

At the second stage, the heating of the considered design of the FOG was modeled under zero gravity conditions and vacuum in outside and inside of FOG (2nd thermal mode).

Graphs of current temperatures and temperature gradients in different elements of the FOG, based on the results of mathematical modeling of the 2 nd thermal regime, are shown in Figure 4.

As we can see from Figure 4, the maximum overheating of the device elements over the medium temperature does not exceed $\Delta T_{\max }=10^{\circ} \mathrm{C}$ in vacuum and zero gravity conditions. These superheats are in 2.5 times higher than those obtained under normal pressure and gravity.

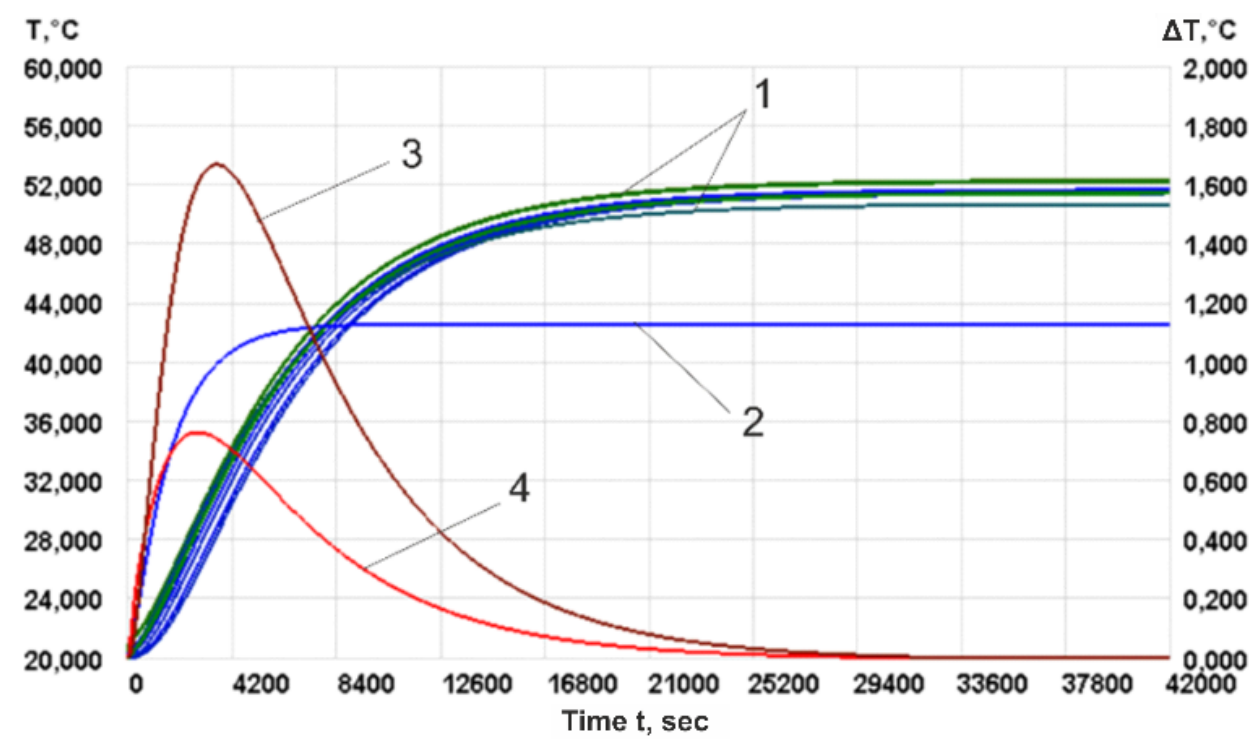

Fig. 4. Current temperatures $T_{i}$ in all FOG elements and и temperature circumferential $\Delta T_{\psi}$ and radial $\Delta T_{R}$ gradients in fiber-optic in case of vacuum and weightless. 1 - temperature of the elements $T_{i}$ of the FOG; 2 - environmental temperature; 3 - circumferential temperature gradient in fiber-optic coil $\Delta T \psi ; 4$ - radial gradient of the temperature in fiber-optic coil of the FOG $\Delta T_{R}$.

The maximum circumferential temperature gradients in the zone of the optical fiber coil of the FOG take values at the level of $\Delta T_{\psi}=1,65^{\circ} \mathrm{C}$, which is slightly lower than the values of these gradients obtained under normal pressure and gravity.

Thus, in the conditions of vacuum and weightlessness, the temperature situation in the FOG worsened in comparison with the conditions of normal atmospheric pressure and the presence of gravity. It is characterized by extended transients (the time of transients increased 
by about 3 times), as well as enlargement overheats over the environment temperature in comparison with the 1-st thermal mode.

The main temperature characteristics of the 1-st and 2-nd thermal modes under constant temperature influence are shown in table 1.

Table 1. FOG temperature characteristics in the 1-st and 2-nd thermal modes.

\begin{tabular}{|c|c|c|c|c|}
\hline $\begin{array}{c}\text { № } \\
\text { mode }\end{array}$ & $\begin{array}{c}\text { Environmental } \\
\text { pressure and gravity }\end{array}$ & $\Delta \boldsymbol{T}_{\boldsymbol{\psi}},{ }^{\mathbf{o}} \mathbf{C}$ & $\Delta \boldsymbol{T}_{\boldsymbol{R}},{ }^{\mathbf{o}} \mathbf{C}$ & $\Delta \boldsymbol{T}_{\boldsymbol{m a x}},{ }^{\mathbf{o}} \mathbf{C}$ \\
\hline 1 & Normal & 1.9 & 1.2 & 4 \\
\hline 2 & Vacuum, zero-gravity & 1.65 & 0.77 & 10 \\
\hline
\end{tabular}

\subsection{Non-stationary temperature disturbances}

In the thermal modes considered above, thermal processes in the FOG were modeled at a constant temperature of the environment. However, in real conditions of orbital flight, the temperature of the media and FOG can change over time, for example, according to the harmonic law [4-5]. Therefore, it is necessary to study how non-stationary external temperature disturbances will affect the temperature fields in the FOG.

The simulation was performed for a period of harmonic influence $\tau=2 \pi / \omega=300,90,30$ and 9 minutes. Medium fluctuations occur in the range from -10 to $+40{ }^{\circ} \mathrm{C}$. Input data on the external temperature effect on the FOG are also obtained by modeling these modes at the upper level (the entire measuring unit).

Graphs of the temperature in all FOG's elements, based on the results of mathematical modeling, are shown in Figure 5.

As we can see, with a harmonic external temperature, the thermal processes in the FOG are also have harmonic nature. Moreover, in the steady process, the temperature in FOG's elements follow up the ambient temperature with the same period but with a certain delay (Figure 5a, b).

It is important to note that when the period of oscillation of the external temperature disturbance decreases, the thermal processes in the FOG change qualitatively and quantitatively.

With a significant reduction of the oscillation period of the external temperature, the temperature in the FOG and temperature gradients are averaged and approximated some steady-state values according to the exponential law (Figure 5c, d).

In this case, the maximum values of the oscillation amplitude are significantly less $\left(\approx 1,4^{\circ} \mathrm{C}\right.$ by $\tau=90 \mathrm{~min}$ and almost zero at $\left.\tau=9 \mathrm{~min}\right)$, than the maximum oscillation amplitudes $\left(\approx 12^{\circ} \mathrm{C}\right)$ for large periods of external temperature disturbances.

It should be noted that the radial $\Delta T_{R}$ and circumferential $\Delta T \psi$ temperature drops in fiber coil also have a harmonic nature and occur near zero in a stady-state mode. The amplitudes of these oscillations for a longer period of external harmonic perturbation (300 $\mathrm{min}$.) amount $\Delta T_{R} \approx 0,8^{\circ} \mathrm{C}, \Delta T \psi \approx 1,8^{\circ} \mathrm{C}$. $\mathrm{C}$ as the period decreases, the values of the different amplitudes decrease and gradually equalize. For 30 minutes, the oscillation amplitudes of the radial and circumferential gradients are approximately equal and are to about $0,1^{\circ} \mathrm{C}$ (Figure $5 \mathrm{c}$ ). With a further reduction of the period of oscillation in the ambient temperature, the radial, and circumferential gradients tend to zero in the steady-state (Figure 5d).

Thus, the harmonically changing external temperature with the considered characteristics not only does not worsen the temperature situation in the FOG but also improves it at relatively high frequencies of external temperature oscillations. At the same time, the nature of thermal processes changes from harmonic to an exponential with a reduction of ambient temperatures amplitude and temperature gradients in the device elements. 
However, in real conditions of orbital flight, the period of temperature oscillation can be quite large, therefore, there may be gradients of temperature fields, which can lead to thermal drift. Therefore, it is advisable to provide measures to reduce the influence of temperature disturbances, for example, by applying algorithmic compensation, which is actively used in gyroscopes of this type, including in the device under consideration, as well as temperature stabilization of either the entire FOG or its individual elements - the fiber coil and the radiator [6-7].

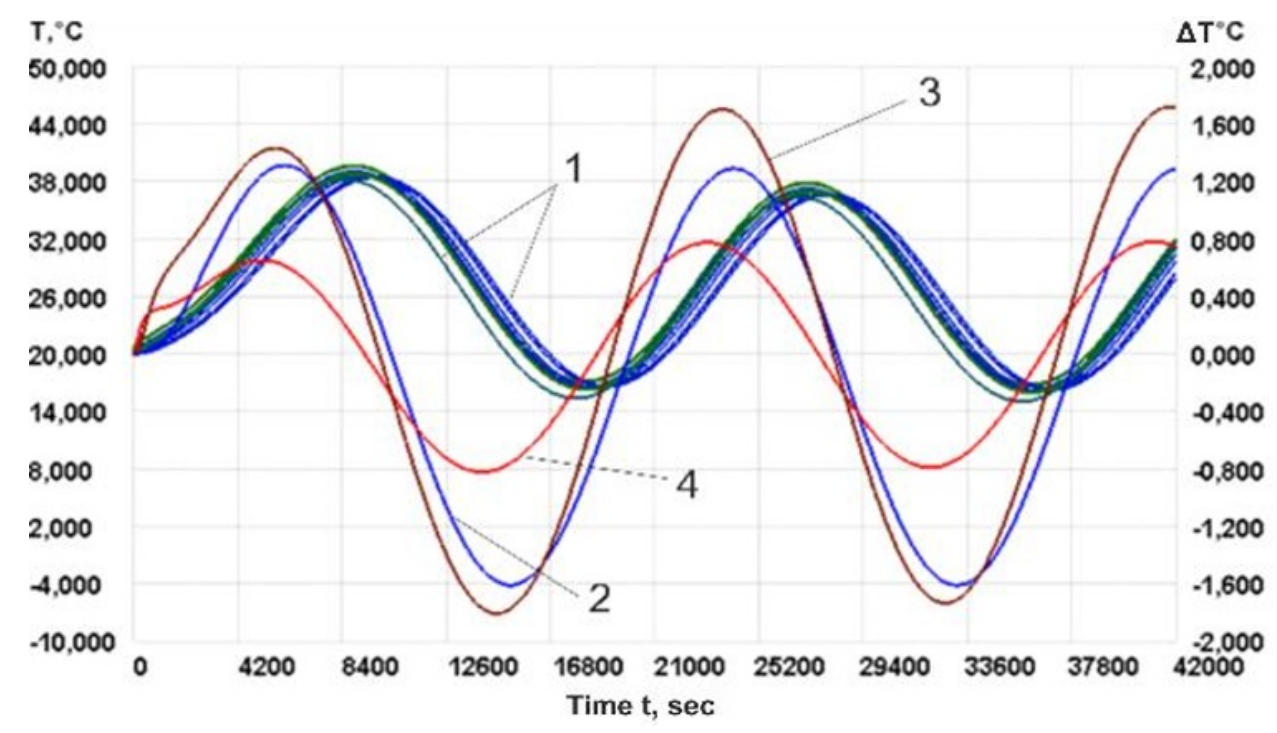

a)



b) 




c)

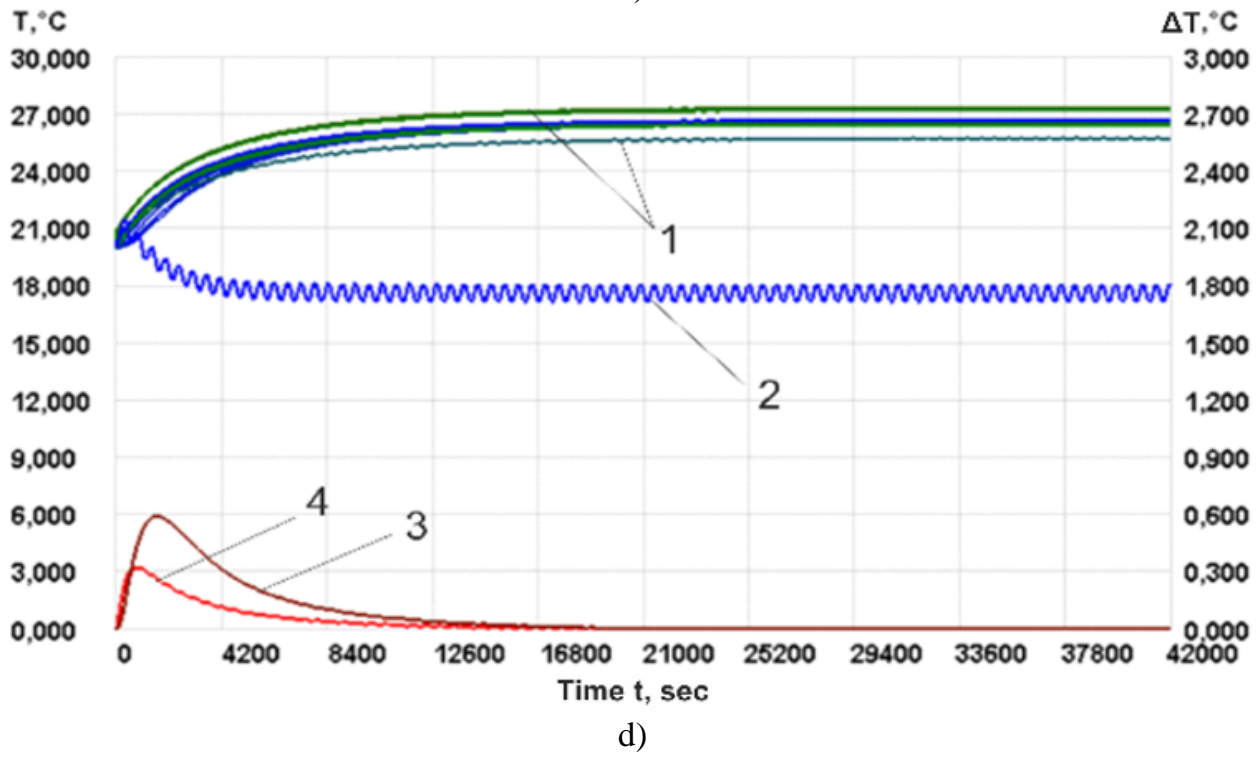

Fig. 5. Temperatures $T_{i}$ at FOG's elements and temperature circumferential $\Delta T_{\psi}$ and radial $\Delta T_{R}$ gradients in the fiber-optic structure under harmonic external temperature $T_{\mathrm{c}}$, in case of vacuum and zero-gravity: a) period $\tau=300 \mathrm{~min}$; б) period $\tau=90 \mathrm{~min}$; c) period $\tau=30 \mathrm{~min}$; d) period $\tau=9 \min$; 1 - the temperature of the elements $T_{i}(1-82)$ of the VOG; 2 - environmental temperature; 3 circumferential temperature drop in fiber coil of the FOG $\Delta T \psi ; 4$ - radial temperature drop in fiber coil of the FOG $\Delta T_{R}$.

\section{Conclusion}

Qualitative and quantitative estimates of the effect of vacuum and zero-gravity conditions, different orientation of the device relative to gravity direction, and the harmonic nature of changes in ambient temperature on the temperature situation in the FOG are obtained.

It was found that in various operating modes in the accepted range of ambient temperature, including in outer space, the maximum overheating of the FOG elements does 
not exceed critical values. It is shown that in the conditions of vacuum and zero-gravity, the temperature situation in the FOG slightly worsens in comparison with the conditions of normal atmospheric pressure and gravity.

Analysis of the thermal situation for the FOG orientation relative to the gravity vector showed a weak dependence under normal atmospheric pressure and gravity conditions. Changes in temperature indicators are within $2 \%$.

It is also revealed that the harmonically varying external temperature with the considered characteristics improves the temperature situation in the FOG at certain frequencies of external temperature oscillation.

In general, these studies have shown that the thermal situation in the FOG is satisfactory, but to increase the thermal invariance and reduce the time of the readiness of the device, it is recommended to provide active measures, for example, the use of reverse temperature control systems.

Thus, the paper shows that the method described in the first part of this paper is suitable for practical application for analyzing three-dimensional non-stationary fields inside gyroscopes as part of space navigation systems.

\section{Acknowledgements}

The work was supported by the RFBR grant 19-08-00839.

\section{References}

1. Djashitov V, Pankratov V 2005 Sensors, devices and systems of aerospace and marine instrumentation in the conditions of thermal effects (St.-Petersburg: SSC RF Central research Institute "Electropribor") p 404

2. Barulina M, Golikov A, Pankratov V, Efremov M 2018 Scientific instrument engineering 28(3) 14

3. Dulnev G, Parfenov V, Sigalov A 1990 Methods for calculating the thermal regime of devices (M.: Radio and communication p 312

4. Golikov A, Pankratov, Barulina M, Pankratova E, Efremof M 2019 Innovations and investments 11167

5. Golikov A V, Pankratov V M and Efremov M V 2018 Gyroscopy and Navigation 9(2) 116

6. Ilyukhin A V, et al 2019 IOP Conference Series: Materials Science and Engineering 643 012102. doi:10.1088/1757-899x/643/1/012102

7. Gorodnichev M G, et al 2019 Systems of Signal Synchronization, Generating and Processing in Telecommunications doi:10.1109/synchroinfo.2019.8814120

(SYNCHROINFO) 